\title{
DISEÑO DE ALCANTARILLA PARA EL PASO DE ORGANISMOS ACUÁTICOS: UN ENFOQUE AMBIENTAL
}

\section{CULVERT DESIGN FOR AQUATIC ORGANISM PASSAGE: AN ENVIROMENTAL APPROACH}

Miguel Enrique Blanco Chávez ${ }^{1}$

(recibido/received: 6-octubre-2020; aceptado/accepted:10-febrero-2021)

RESUMEN: El diseño de las alcantarillas en el cruce de carreteras y corrientes de agua como quebradas o ríos, se ha basado tradicionalmente en los requisitos hidráulicos de seguridad para la carretera. Con esta concepción del diseño de alcantarilla se ha dejado sin consideración los elementos biológicos de la corriente de agua, tal como los peces y otros organismos acuáticos, los cuales son parte esencial del ecosistema y que ha sido alterado por una barrera física que es la alcantarilla. Incluso, algunos factores físicos también son alterados, como la erosión o deposición de sedimentos y detritos, los cuales cambian igualmente las condiciones ambientales para los peces, dando como resultado, barreras que le impiden el desarrollo natural de su ciclo de vida, y ponen en peligro su reproducción. Pensando en el diseño amigable con el medio ambiente, se han desarrollado conceptos y lineamientos para el diseño de alcantarillas de cruce de carreteras y corrientes de agua, que faciliten el pase de los organismos acuáticos a través de la alcantarilla, de modo que estos puedan tener movilidad de la misma forma que en la corriente natural. En este escrito se presentan algunos lineamientos para el diseño de alcantarillas de forma amigable con el medio ambiente, los que son abordados por Kilgore et al (2010), Schall at al. (2012), Bates y Kirn (2009), Henrik et al (2019), Kozarek y Mielke (2015), Olson et al (2017) y Departamento de Pesca y Vida Silvestre de Vermont (2016).

PALABRAS CLAVE: Ríos, alcantarilla, paso de peces, ambiente.

${ }^{1}$ Docente del Programa de Investigación, Estudios Nacionales y Servicios del Ambiente (PIENSA-UNI). Managua, Nicaragua. Correo electrónico: meblanco_ch@yahoo.es. ORCID:0000-002-3138-4209 
ABSTRACT: The design of culverts at the crossroads of roads and streams such as streams or rivers, has traditionally been based on the hydraulic safety requirements for the road. With this conception of the sewer design, the biological elements of the water stream, such as fish and other aquatic organisms, which are an essential part of the ecosystem and which have been altered by a physical barrier that is the sewer, have been left without consideration. Thinking about environmentally friendly design, concepts and guidelines have been developed for the design of culverts to cross roads and streams, which facilitate the passage of aquatic organisms through the culvert, so that these can have mobility in the same way as in the natural stream. In this paper some guidelines for the design of culverts in an environmentally friendly way are presented, which are addressed by Kilgore et al (2010), Schall at al. (2012), Bates and Kirn (2009), Henrik et al (2019), Kozarek and Mielke (2015), Olson et al (2017), and Vermont Department of Fish and Wildlife (2016).

KEYWORDS: Rivers, culvert, fish passage, environmental.

\section{INTRODUCCIÓN}

En la práctica tradicional del diseño de alcantarillas en el cruce de carreteras y quebradas o ríos, se utilizan criterios hidráulicos como carga hidráulica a la entrada de la alcantarilla, las velocidades de aproximación y de salida de la alcantarilla, y la relación carga-diámetro (HW/D), de forma que aseguren el paso del caudal de diseño sin que afecte la carretera estructuralmente y funcionalmente. Estos criterios han resultado bastante prácticos desde el punto de vista ingenieril, lográndose desarrollar diseños bastante eficientes. Se han incluido desde luego la alineación adecuada con respecto a la dirección de la corriente y los tipos de material del fondo y taludes del cauce, para evitar procesos de erosión, gradación e inundaciones no deseadas a la propiedad adyacente. Sin embargo, muy poco se ha considerado en este enfoque del diseño de alcantarilla, los organismos acuáticos que dependen del ambiente y su preservación, de manera que puedan vivir sin riesgo de extinción. Así, la construcción de alcantarillas en algunas ocasiones podría afectar las condiciones ambientales que aseguren su movilidad, reproducción y el ciclo de vida. Cuando se crea una carga hidráulica (HW) aguas arriba de una alcantarilla se cambia la velocidad de la corriente aguas arriba de ella, en el interior de la misma e igual a su salida, o podría haber una caída hidráulica que no sería posible vencer si un pez requiera movilidad aguas arriba. Los investigadores Anderson et al (2014) en un estudio realizado en dos subcuencas de los Condados de Tucker y Randolph, West Virginia, USA, determinaron que el 55\% de los sitios visitados con alcantarillas formaban barreras completas y $34 \%$ formaban barreras parciales para el movimiento de la Salamandra de río, y que al menos, $20 \%$ de la longitud total de las corrientes de las dos sub cuencas estudiadas estaban aisladas por al menos una barrera parcial. El Departamento de Pesca y Vida Silvestre de Vermont (VFWD,2016) condujo un estudio sobre el paso de organismos acuáticos en 1,501 alcantarillas, donde señala que "se encontró que menos del 6\% preveía un paso completo de organismos acuáticos" (p.1). Esto nos da una idea del nivel de afectación que las alcantarillas de cruce de carreteras podrían tener en los ecosistemas de ríos y quebradas. La

El Higo Revista Científica / Volumen 11. No. 01, pp. 1-14/ junio 2021 
difusión de métodos desarrollados para permitir el paso de los organismos acuáticos en alcantarillas contribuye a realizar diseños amigables con el medio ambiente a medida que más profesionales se apropian de este enfoque.

\section{DESARROLLO}

En el diseño del cruce de carretera y alcantarilla, Anderson, P.T., del Servicio Forestal de Estados Unidos de Norteamérica (2011) menciona que "el pase de los organismos acuáticos y la conectividad ecológica es el objetivo y la principal prioridad del diseño para el cruce de una corriente que provee hábitat para la vida acuática" (p. 2-2). Otras consideraciones de diseño que menciona son "minimizar las consecuencias del taponamiento y el rebose, incluyendo evitar la división de la corriente", "proveer suficiente capacidad hidráulica, de forma que la carga hidráulica para el caudal máximo no cause flujo a presión" y "maximizar los beneficios mientras se minimiza el costo de vida del proyecto".

El Ministerio de Agricultura, Alimentación y Medio Ambiente de España (2015), establece medidas preventivas y prescripciones técnicas para asegurar el paso de los peces y otros organismos acuáticos, tales como las estructuras para peces: "Son aplicables las estructuras que permiten mantener el cauce fluvial intacto, básicamente viaductos adaptados, y en algunos casos drenajes adecuadamente acondicionados" (p. 33). Con esto se persiguen los mismos objetivos descritos por el United States Department of Agriculture. Forest Service (USFS) en el párrafo anterior.

En un estudio realizado por la Universidad Tecnológica de Michigan (Olson et al.,2017), los autores encontraron que las alcantarillas de reemplazo instaladas para proveer el paso de organismos acuáticos (POA), diseñadas por el método de simulación de la corriente, mantienen mejor la retención de la materia orgánica particulada gruesa a través de las alcantarillas, y no se observó diferencias en los procesos de los ecosistemas entre los tramos localizados aguas arriba y aguas abajo de las alcantarillas de reemplazo. Esto nos indica que las alcantarillas de reemplazo no tuvieron afectación significativa en los procesos de los ecosistemas en las corrientes estudiadas, pero se deben realizar más estudios de este tipo para profundizar este conocimiento.

El Departamento de Pescas y Vida Silvestre de Vermont, (VFWD, 2016, p. 6), establece que el cruce seguro y estable de corrientes de agua permite el alojamiento de la población acuática y protege la salud de la corriente a la vez que reduce la erosión del cauce y el daño estructural. Algunas consideraciones que hacen para un efectivo diseño de cruces son:

Tipo de estructura: se prefieren las estructuras de fondo abierto tales como arcos y puentes dado que ella mantiene el fondo natural del rio y tienen menos problemas para desarrollar problemas de paso. En muchos casos las estructuras cerradas de cruce de corrientes (con fondo) son aceptables si son diseñadas y construidas apropiadamente. 
Ancho de la estructura: el cruce no deberá disminuir el ancho del agua para la sección llena del canal, no incluye la llanura de inundación.

Longitud de la estructura: estructuras más largas alteran más el hábitat de la corriente y a menudo les es más difícil y frecuente de proveer paso. El uso de estructuras anchas y muros cabezales pueden reducir la longitud de la estructura y minimizar el impacto a la corriente.

Alineamiento con la corriente del canal: el alineamiento de la estructura de cruce con la corriente es una parte esencial del diseño. Cuando la alcantarilla no es apropiadamente alineada con el canal aguas arriba y aguas abajo, se reduce la capacidad hidráulica y se incrementa el taponamiento por sedimentos.

Alineamiento vertical: el perfil del fondo en el cruce deberá en lo posible coincidir con el perfil del fondo del canal natural de la corriente. Este perfil debería determinarse para las condiciones anteriores a la construcción del cruce.

Alcantarilla embebida y fondo del río natural: el substrato de la corriente debería ser en lo posible igual al sustrato natural encontrado en la corriente del canal aguas arriba y abajo del cruce. La alcantarilla debería permanecer embebida, creando una transición suave entre la corriente el canal natural y la estructura de cruce.

Profundidad y Velocidad: el cruce debería ser esencialmente "invisible" para los peces y la vida silvestre. Los cruces deberían ser diseñados para mantener un flujo y substratos adecuados. Para caudales bajos o altos, las profundidades y velocidades del agua deberían ser las mismas como las de la corriente natural aguas arriba y aguas abajo del cruce.

Para diseñar el Paso de los Organismos Acuáticos (POA) se requiere hacer simulación de la corriente del cauce natural del río o quebrada, bajo las condiciones naturales antes de la alcantarilla y en las condiciones con la alcantarilla instalada. La USFS define "la simulación de la corriente es un método de diseño de estructuras de cruce (usualmente alcantarillas), con el objetivo de crear dentro de la estructura un canal tan similar como sea posible al canal natural. La premisa es que el canal simulado no presentaría obstáculo adicional a los que se presentan en el canal natural" (2008, p.3-1).

Bates et al. (2009) presentan tres métodos para el diseño de pase de organismos acuáticos: opción de pendiente baja, opción simulación de la corriente y opción hidráulica.

El método de "pendiente baja" es un método simplificado de diseño que se aplicaría solamente en sitios de bajo riesgo de fondo estable pero móvil, corrientes con pendientes bajas y longitud corta de alcantarilla. La longitud de la alcantarilla está limitada a 15.24 m (50 pies) y la pendiente del canal está limitada a menor del 1\%. El fondo de la alcantarilla se embebe de 20 a $40 \%$ de su altura (diámetro para alcantarilla circular, el equivalente para otras formas) para la elevación del fondo 
esperada en la vida útil del proyecto. La cobertura mínima deberá utilizarse al pie de las estructuras con fondo abierto para el nivel mínimo esperado del fondo del canal. El ancho de la alcantarilla a la elevación donde esta toca el fondo de la corriente debe ser al menos 1.25 veces el acho promedio de la superficie libre del agua para la sección llena del canal principal (bankfull). La alcantarilla no restringe excesivamente la planicie de inundación activa. Este método solo aplica para instalaciones nuevas y donde se requiere el paso de especies acuáticas débiles.

El método de "simulación de la corriente del río" se aplica a nuevas instalaciones de alcantarillas y corrientes con cualquier pendiente. La simulación de la corriente es un método con enfoque geomorfológico para el diseño de paso de peces y organismos acuáticos. Es una continuación de las dimensiones del canal natural, pendiente, material del fondo y bancos a través del cruce para conectar los canales aguas arriba y aguas abajo del cruce. La simulación de la corriente crea las diversas profundidades del agua y velocidades, aéreas de ocultamiento y descanso, y el hábitat húmedo de contorno que diferentes especies necesitan para moverse. El canal simulado en el interior del cruce no presentaría más obstáculos para el movimiento que los obstáculos presentes en el canal natural adyacente.

El método de "diseño hidráulico" es un proceso de diseño que reúne las características hidráulicas de una alcantarilla en un rango específico de caudales con las habilidades de nado de una especie objetivo y una edad especifica del pez. La hidráulica de la alcantarilla podría ser controlada por la pendiente de la alcantarilla, el ancho (o diámetro) y la rugosidad. Este método persigue una especie distintiva de pez, por eso no cuenta para los requerimientos biológicos de especies que no son el objetivo del diseño.

El método de simulación de la corriente también es presentado por la Administración Federal de Autopistas FHWA (Schall et al., 2012) y por el Departamento de Transporte de Minnesota (Hernick, et al.,2019) y se considera el mejor para el diseño del POA, por lo cual lo abordaremos en las siguientes líneas.

\section{Contexto del Paso de Organismos Acuáticos (POA)}

El paso de los organismos acuáticos POA, (AOP por sus siglas en idioma inglés) en alcantarillas se efectúa en un contexto específico que debe ser tomado en cuenta, y que se describe a continuación.

Instalaciones nuevas versus rehabilitación. A pesar de que los objetivos de todos los POA de alcantarillas son los mismos, el facilitar el paso a través de alcantarillas, hay diferencias en el alcance y el rango de opciones para nuevas instalaciones de alcantarillas y rehabilitación de alcantarillas. Las nuevas instalaciones, incluyendo el reemplazo de alcantarillas, proveen la flexibilidad para variar el tipo, tamaño, forma, pendiente, alineamiento, y el material del fondo dentro de la alcantarilla para satisfacer los objetivos POA. Las opciones pueden incluir alcantarillas con 
fondo abierto y alcantarillas con fondo cerrado con el invertido embebido debajo de la cama del arroyo o corriente.

Paso de barreras. El diseño de alcantarillas sin considerar los organismos acuáticos podría crear barreras para el movimiento aguas arriba y abajo de la corriente. Los organismos necesitan la movilidad para buscar alimento, encontrar refugio, evitar los predadores o reproducirse. Una alcantarilla llega a ser una barrera para los POA cuando esta posee condiciones que exceden las capacidades físicas del organismo. Las circunstancias que sirven como barreras dependen de las especies y la etapa de vida (joven o adulto) del organismo. Para un pez, el enfoque de mayores obstrucciones comunes de AOP incluye:

Excesiva velocidad de agua. Caídas en la entrada o salida de la alcantarilla (ver Figura 1). Barreras físicas tales como vertederos, pantallas, o escombros en el conducto de la alcantarilla. Turbulencia excesiva causada por concentración o expansión del flujo. Flujos bajos que proveen muy poca profundidad para que el pez nade (figura 1).
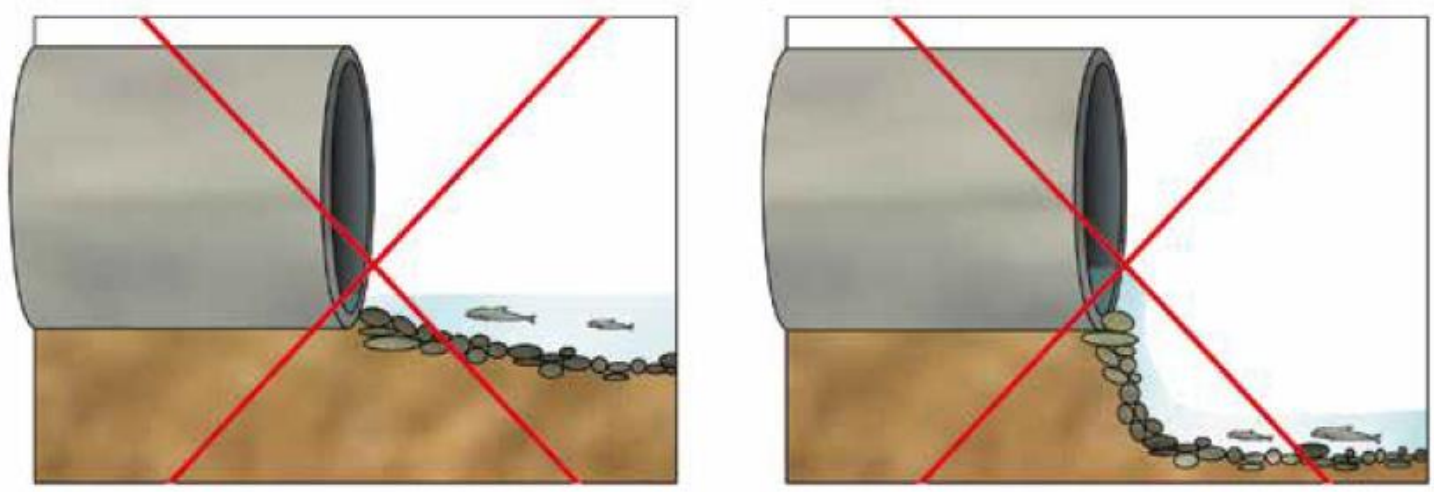

Figura 1. Dos alternativas que dificultan el remonte de los peces debido a la poca altura de la lámina de agua o al importante desnivel (tomado del Ministerio de Agricultura, Alimentación y Medio Ambiente de España, 2015).

Biología del pez. Las capacidades físicas del pez dependen de dos sistemas de músculos que tratan de acomodar diferentes modos de viaje: un sistema de músculos rojo (aeróbico) para actividades de baja intensidad y un sistema de músculos blancos (anaeróbico) para movimientos cortos de alta intensidad. Un pez que llega a estar exhausto ya sea en actividad de alta intensidad o baja intensidad requerirá un periodo de reposo antes de continuar moviéndose. El uso extensivo del sistema de músculos blancos para movimientos de alta intensidad, en particular, causa fatiga extrema, requiriendo períodos extendidos de reposo.

El pez puede fallar el paso de una alcantarilla por varias razones, pero entre las razones más comunes están las barreras que requieren la actividad del sistema de músculos en exceso de la capacidad física. Una salida con caída o una zona con alta velocidad actuaría como una barrera cuando esta excede la habilidad de nado emergido del pez, mientras que una sección continua 
larga de la alcantarilla con velocidad relativamente baja podría requerir prolongadas velocidades de nado a ser mantenidas más allá de la habilidad natural del pez.

La etapa de la vida también es importante. Un diseño que reúna las necesidades de un salmón adulto desovando, por ejemplo, no necesariamente garantizará que una alcantarilla permitirá el paso de un salmón joven más débil. A demás, a pesar de que el pez podría ser capaz de tener una energía especifica de nado, esto no significa que el pez elegirá el gastar la máxima energía de nado cuando enfrente determinado obstáculo. En cambio, el pez ha sido también observado buscando regiones de profundidades mayores con velocidades más bajas dentro de la compleja dinámica del flujo en una alcantarilla para llegar al paso.

Hidrología. Los requerimientos de los organismos acuáticos varían de especie a especie y con la etapa de la vida, pero a menudo están relacionados a las estaciones y las condiciones del flujo. Para un pez, la alcantarilla debería permitir el paso para un rango de flujos correspondientes al tiempo y extensión del movimiento del pez dentro del canal del arroyo. En un tramo de corriente natural, el pez responde a eventos de alto caudal buscando refugio, hasta que las condiciones del canal acaben. Durante los extremos de bajo caudal, las profundidades bajas pueden causar que el mismo canal por sí sólo se vuelva no pasable. Generalmente, los límites altos y bajos limitan las condiciones del flujo en la cual el paso debe ser proveído y éstos son referidos como flujos de paso altos y bajos.

Flujos de paso alto, QH, representan el límite superior de caudal para el cual se cree que el pez está en movimiento dentro de la corriente, mientras el caudal de pase bajo, QL, es el caudal más bajo requerido para el paso del pez, generalmente basado en la mínima profundidad de flujo requerido para el paso del pez. Los caudales de diseño de paso alto y bajo no son definidos de la misma manera en el país. La variación puede reflejar las diferencias en hidrología y las especies de región a región, pero podría reflejar también inconsistencias en la definición de estos términos.

Geomorfología y estabilidad de la corriente. Como una estructura rígida en un ambiente dinámico, todas las alcantarillas deben ser diseñadas teniendo en mente que un canal está en proceso de formación. Diseños efectivos consideran el canal y la cuenca en el contexto de la localización del cruce. Los canales están continuamente cambiando, y el potencial del ajuste del canal debe ser entendido. Sin una consideración apropiada, planes bien intencionados podrían en detrimento afectar el sistema del arroyo y el hábitat relacionado. Por ejemplo, si la erosión esta en progreso aguas arriba de la localización de la alcantarilla y esta no es ni identificada ni mitigada, esa inestabilidad eventualmente alcanzará el sitio. Dependiendo del tipo de instalación de alcantarilla, la erosión podría resultar en una caída a la salida de la alcantarilla, desestabilización de la alcantarilla o ambas.

En un estudio realizado por los investigadores Kozarek et al. (2015) en la Universidad de Minnesota, en el que evalúan la influencia del relleno en una alcantarilla de cajón embebida como parte del proceso de instalación en corrientes con pendientes bajas, medias y moderadas, 
encontraron que: "En general, el rellenar la alcantarilla resulta en menos riesgo de erosión aguas arriba de la misma. Colocar material del fondo del río dentro de la alcantarilla durante el proceso de instalación también asegura que el sedimento permanezca en la alcantarilla con el caudal para sección llena del canal (bankfull) y con un hidrograma de tormenta simulado" (p. 1). Esta relevante conclusión, aunque no definitiva, respalda la importancia de la geomorfología en la simulación de la corriente para el diseño del POA.

\section{Procedimiento de diseño para nuevas instalaciones}

Instalaciones nuevas de alcantarillas o de reemplazos permiten un diseño POA para considerar el rango total de las características de las alcantarillas en el diseño incluyendo el tipo, tamaño, pendiente, alineamiento y material del fondo dentro de la alcantarilla. El estado del arte en el diseño de un POA es el uso de simulación de la corriente (Schall et al., 2012). Esto es, por la aplicación de la técnica de simulación de la corriente, el diseñador trata de simular las condiciones de la corriente natural dentro de la alcantarilla de manera que los organismos acuáticos puedan moverse a través de la alcantarilla como también podrían ser capaces de moverse a través de la corriente para aquellas condiciones más críticas para el organismo.

A continuación, en esta sección se aborda someramente el procedimiento de simulación de corrientes como se describe en FHWAHEC 26 (Kilgore et al., 2010). Esta metodología aplica a alcantarillas con fondo cerrado embebido y alcantarillas con el fondo abierto. En cualquiera de los casos, el material natural en el fondo es proveído dentro de la alcantarilla como se muestra en el esquema de la Figura 2.

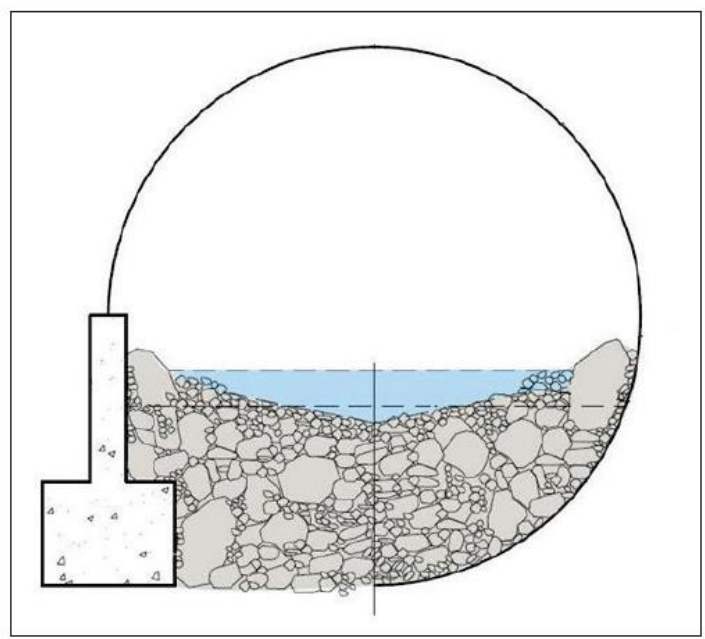

Figura 2. Embebido para el fondo abierto o cerrado de la alcantarilla (adaptado de USFS, 2008).

Debido a la amplia variedad de organismos acuáticos que podrían ser relevantes para un sitio en particular, los procedimientos de simulación de arroyos tratan de proveer el paso sin necesidad de analizar el comportamiento de ninguna especie en particular u objetivo. Para completar esto, medidas sustitutas han sido desarrolladas para cada método de simulación de corriente para guiar

El Higo Revista Científica / Volumen 11. No. 01, pp. 1-14/ junio 2021 
al diseñador. La aplicación de los métodos de diseño de simulación de corrientes es a menudo mejor completada por un equipo interdisciplinario de biólogos acuáticos, geomorfologos e ingenieros.

Medidas sustitutas. Dado el diverso comportamiento y capacidades de los peces y otros organismos acuáticos, los procedimientos de diseño necesariamente dependen de parámetros sustitutos e indicadores como medidas para un exitoso diseño del paso.

El procedimiento del FHWA HEC 26 (Kilgore et al., 2010) utiliza el comportamiento del sedimento como su parámetro sustituto. La hipótesis para utilizar el comportamiento del sedimento como parámetro sustituto es que los organismos acuáticos en un arroyo están expuestos a similares fuerzas y esfuerzos experimentados por el material del fondo. Por eso, si el diseño de la alcantarilla no altera las fuerzas en el fondo del canal, entonces se puede presumir que no altera las fuerzas experimentadas por los organismos acuáticos. El objetivo del diseño es proveer un cruce de arroyo que tenga un efecto equivalente, en un rango de flujos de la corriente, en el material del fondo dentro de la alcantarilla comparado con el material del fondo del arroyo aguas arriba y aguas abajo de la alcantarilla.

En el caso de ambos criterios sustitutos, cuando los criterios sustitutos son satisfactorios, la hipótesis es que las condiciones a través de la alcantarilla no deberían presentar más obstáculo a los organismos acuáticos que las condiciones en el canal natural adyacente. A pesar de que no hay requerimiento para evaluar requerimientos biológicos específicos en un sitio dado, tal información de especies específicas puede ser incorporada en el proceso de diseño de la simulación del arroyo cuando está disponible. Una instalación completa de alcantarilla con fondo abierta en un arroyo se muestra en la Figura 3.

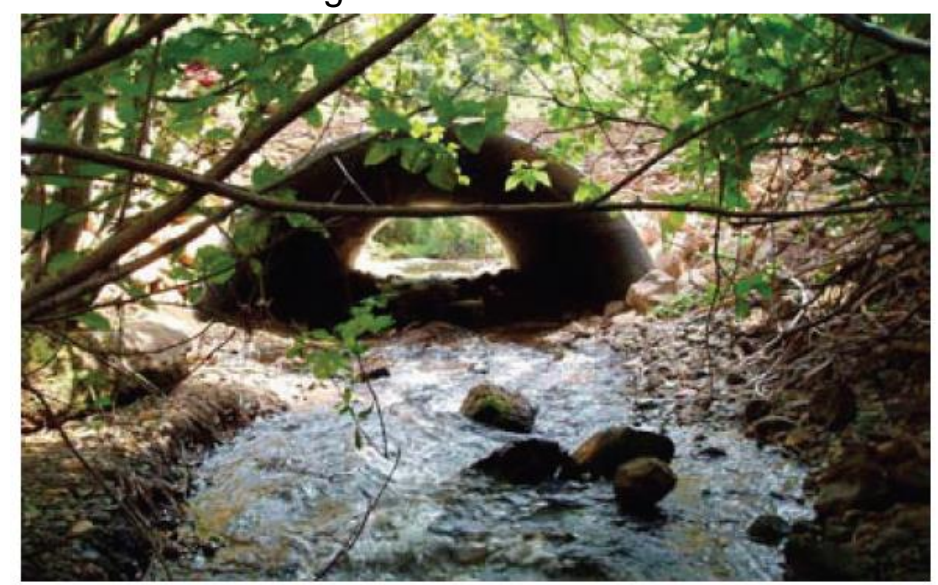

Figura 3. Instalación completa de alcantarilla con fondo abierto (tomado de USFS, 2008).

Simulación del flujo del FHWA HEC 26. El procedimiento de diseño de simulación del flujo en el FHWA HEC 26 (Kilgore et al., 2010) fue desarrollado en base a la medida sustituta de estabilidad del fondo más que en el ancho con sección llena(bankfull) para abordar las limitaciones en la estimación de un apropiado ancho con sección llena en muchas situaciones. También fue 
desarrollado para proveer un caso reproducible basado en las mejores prácticas para soportar las decisiones de diseño que a menudo resultan en mayores, y por eso, costos de capital más caros para proveer una POA.

El proceso de diseño se enfoca en cinco variables primarias que deberán ser determinadas para cada sitio.

1. Flujo pico de diseño, Qp. Este caudal puede ser el $Q_{25}, Q_{50} \circ Q_{100}$ requerido por el sitio para direccionar los caudales de diseño de crecida. Este es el caudal tradicionalmente utilizado para diseñar una alcantarilla $(\mathrm{Qx}$ representan el caudal del X-año de intervalo de recurrencia).

2. Caudal de diseño de paso alto, QH. Este es el caudal máximo utilizado para el diseño del paso. Este puede aplicar para todo el año o para una estación específica.

3. Caudal de diseño de paso bajo, QL. Este es el caudal mínimo utilizado para el diseño del paso. Este puede aplicar para todo el año o para una estación específica.

4. Características del material del fondo. Para materiales no cohesivos, se requieren las propiedades características del fondo, incluyen $D_{16}, D_{50}, D_{84}$.

5. Esfuerzo cortante permisible, $\mathrm{tp}$, del material del fondo.

Cinco pruebas fundamentales son aplicadas como parte del procedimiento. Si cualquier prueba falla, ajustes de diseño se especifican. Las pruebas son:

1. ¿Satisface la alcantarilla los requerimientos de caudal pico, Qp?

2. ¿Es el material del fondo en la alcantarilla estable (no se mueve o el sedimento entrante es igual al saliente) para el caudal de paso alto de diseño, QH?

3. ¿Es el material del fondo en la alcantarilla estable para el caudal pico de diseño, Qp? (una capa de anclaje / accesorio debajo del material del fondo podría requerirse para satisfacer esta prueba).

4. ¿Es la velocidad en la alcantarilla para el caudal de diseño de paso alto, QH, consistente con las velocidades de aguas arriba y aguas abajo del canal?

5. ¿Es la profundidad en la alcantarilla para el caudal de diseño de paso bajo, QL, consistente con las profundidades de aguas arriba y aguas abajo del canal?

El primer ítem es la prueba tradicional para la adecuación hidráulica. Todos los criterios y requerimientos para la suficiencia hidráulica aplican cuando se diseña para el POA, pero usualmente no son el factor limitante en el diseño.

La segunda prueba es el uso inicial de la estabilidad del fondo del canal sustituto. Las instalaciones de alcantarillas nuevas POA y de reemplazo tendrán un fondo natural dentro de la alcantarilla ya sea con el uso de alcantarilla de fondo abierto o alcantarilla con fondo cerrado y embebido. Es deseable que el fondo del material del arroyo sea estable con el caudal de paso alto, aunque no podrá ser estable para algunos materiales, particularmente en un canal de fondo de arena. El 
proceso de diseño provee un marco de trabajo para determinar cuándo un diseño satisface esta prueba.

La tercera prueba también examina la estabilidad del fondo sustituto, pero con el caudal pico de diseño. Esta prueba direcciona el desempeño de larga duración del material del fondo del arroyo, especialmente para una alcantarilla embebida. Con el caudal pico, algunos materiales podrían ser lavados fuera de la alcantarilla, sin embargo, en algunos casos este podría ser rellenado en la rama descendente del hidrograma de crecida cuando el material es re depositado. En muchos casos, una capa acorazada sobredimensionada es recomendada para evitar exponer el invertido de la alcantarilla y para mejorar el proceso de re depositado.

La cuarta y quinta prueba examina las velocidades para QH y las profundidades estimadas para QL en la alcantarilla y en los tramos del canal aguas arriba y aguas abajo. Si ambos están dentro del rango observado en el flujo natural de la corriente, es razonable concluir que, si un organismo acuático puede sortear el canal natural, este también puede transitar a través de la alcantarilla. Esta comparación es entre las condiciones ambientales en el arroyo y las condiciones ambientales de la alcantarilla, no es con la habilidad de cualquier pez particular u otras especies de organismos acuáticos.

Si cualquiera de las pruebas falla, el procedimiento de diseño ofrece guía de cómo cambiar el diseño de la alcantarilla para satisfacer las cinco pruebas. El procedimiento confía en las mejores prácticas en la estimación de las propiedades del fondo del canal incluyendo el esfuerzo cortante permisible y el coeficiente de rugosidad de Manning. A medida que se aumenta el entendimiento técnico de esos procesos, el mismo marco de trabajo es aplicado, pero las prácticas mejoradas pueden ser incorporadas en el método.

\section{Opciones de restauración de alcantarillas}

En ocasiones se requiere restaurar alcantarillas existentes para facilitar el POA cuando su reemplazo no es factible por razones constructivas o de costos. En este caso el tamaño y el material de la alcantarilla están predeterminadas, así como su alineamiento horizontal y vertical. El enfoque de la restauración generalmente cambia a comparar la condición hidráulica (velocidad y profundidad) dentro de la alcantarilla a la capacidad de movimiento de determinadas especies, generalmente peces.

Las pantallas, bloques materiales del fondo grande son algunas de las técnicas que pueden ser utilizadas dentro de la alcantarilla existente para disminuir la velocidad, incrementar la profundidad del flujo y proveer más variación hidráulica, de manera que un pez en particular en una etapa de vida especifica en una estación del año dada pueda ser capaz de pasar por la alcantarilla. Sin embargo, la capacidad de la alcantarilla restaurada debe ser revisada para el caudal máximo de crecida, para verificar que su capacidad no se ha reducido de forma no aceptable al agregarle uno o más de estos elementos. 
El diseñador debe reconocer que la restauración de la alcantarilla para fines POA involucra los compromisos de intentar proveer unas mejorías aceptables para el POA mientras se mantiene la capacidad hidráulica suficiente. Al igual que con el diseño de alcantarillas nuevas y de reemplazos, un equipo multidisciplinario de biólogos e ingenieros deben trabajar juntos para encontrar el balance apropiado en un sitio en particular.

\section{CONCLUSIONES}

El desarrollo del enfoque ambiental del diseño de carreteras para garantizar la conectividad ecológica en las corrientes de agua, incluye el dimensionamiento de las estructuras hidráulicas de cruce de corrientes que soportan vida acuática.

El planteamiento común en los documentos de los autores consultados, es que en el diseño del paso de organismos acuáticos (AOP), la alcantarilla deberá tener como mínimo el ancho de la sección del canal de la corriente con el caudal a sección llena, aproximadamente la misma pendiente longitudinal y velocidad del flujo del canal antes de la instalación, o similares al tramo del canal aguas arriba y aguas a abajo del sitio de la instalación, que la alcantarilla sea de fondo abierto o embebido y que el material del fondo de la misma conserve las mismas características granulométricas. Con estas condiciones del diseño del APO se pretende que el comportamiento hidráulico en el interior de la alcantarilla sea similar al del cauce natural de la corriente, y por tanto los organismos acuáticos no encontrarían dificultades adicionales para su desplazamiento en el interior de la misma, así como aguas arriba y aguas abajo del paso AOP.

Otro aspecto importante con respecto al material del fondo del AOP, como lo resalta Kozarek et al. (2015), es que este material se tome del material del fondo de la corriente y sea instalado en el momento de construcción de la alcantarilla, dado que si no se hace de esta forma, para que la corriente rellene el fondo con material de su cauce, en corrientes con pendiente baja, esto podría tomar mucho tiempo, en algunos casos años, y no se cumpliría el objetivo planteado de no alterar las condiciones ambientales en la alcantarilla.

Si bien hay diversos métodos de diseño del AOP, se considera que el método de simulación de la corriente es el más adecuado tal como lo plantean Schall et al. (2012), (Olson et al.,2017) y Hernick, et al. (2019), ya que este es aplicable a corrientes con diferentes pendientes, toma en cuenta las características geomorfológicas del sitio de estudio e hidrológicas de la corriente, y hace uso de las técnicas matemáticas modernas de simulación, facilitando el análisis de alternativas de solución.

Tal como lo plantean los autores Schall et al. (2012), se requiere la participación de profesionales de diferentes disciplinas para la determinación de los parámetros de diseño de este nuevo enfoque de diseño de la alcantarilla, especialmente de biólogos acuáticos, geomorfologos, ingenieros hidrólogos e hidráulicos. El estudio de la biología de las diferentes especies acuáticas de las

El Higo Revista Científica / Volumen 11. No. 01, pp. 1-14/ junio 2021 
corrientes para las diferentes regiones del país es un tema de investigación necesario para poder aplicar la metodología de diseño de alcantarilla abordado.

Si bien las alcantarillas con enfoque AOP resultan con mayores dimensiones que las diseñadas únicamente con el criterio hidráulico, incrementando los costos del proyecto de carreteras, las primeras tienen un mejor desempeño en capacidad y en funcionamiento, a la vez que son amigables con el ambiente acuático.

Las nuevas técnicas de simulación hidrológica e hidráulica permiten aplicar las metodologías de diseño de alcantarillas que facilitan el Paso de Organismos Acuáticos (POA), que contribuyen a preservar la biodiversidad acuática de las corrientes de agua que cruzan las carreteras. La apropiación y aplicación de estos conocimientos por parte de los profesionales ambientalistas permitirá alcanzar los objetivos planteados.

\section{REFERENCIAS}

Anderson, J. T., Ward, L.R., Todd, J., Kite, J. S., y Strager, M.P. (2014). Culvert Effects on Stream and Stream-Side Salamander Habitats. International Journal of Environmental Science and Development, Vol. 5, No. 3.DOI: 10.7763/IJESD. 2014.V5.491

Anderson, P.T. (2011). Design for Aquatic Organism Passage at Road-Stream Crossings: Simple Stream Simulation Example. USDA Forest Service. https://www.dirtandgravel.psu.edu/sites/default/files/Education\%20Training/workshops/2012/ documents/AOP.pdf

Bates, K. K. y Kirn, R. (2009). Aquatic Organism Passage Design and Stream Assessments: Guidelines for the Design of Stream/Road Crossings for Passage of Aquatic Organisms in Vermont. Vermont Department of Fish y Wildlife. https://vtfishandwildlife.com/sites/fishandwildlife/files/documents/Learn\%20More/Library/REP ORTS\%20AND\%20DOCUMENTS/AOP/GUIDESLINES\%20FOR\%20DESIGN.pdf

Hernick, M.,Lenhart, C.,Kozarek, J.yNieber, J. (2019).Minnesota Guide for Stream Connectivity and Aquatic Organism Passage through Culverts. Final Report.University of Minnesota.https://www.dot.state.mn.us/research/reports/2019/201902.pdf

Kilgore, R. T., Bergendahl, B. S., y Hotchkiss, R. (2010). Culvert design for Aquatic Organism Passage. Hydraulic Engineering Circular Number 26 (HEC-26).FHWA HIF-11-008. Federal Highway Administration https://www.fhwa.dot.gov/engineering/hydraulics/pubs/11008/hif11008.pdf

(FHWA).

Kozarek, J. y Mielke, S. (2015). Sediment Transport through Recessed Culverts: Laboratory Experiments Final Report.University of Minnesota. https://www.Irrb.org/pdf/201508.pdf 
Ministerio de Agricultura, Alimentación y Medio Ambiente (2015). Prescripciones técnicas para el diseño de pasos de fauna y vallados perimetrales (2da ed., revisada y ampliada).

Documentos para la reducción de la fragmentación de hábitats causada por infraestructuras de transportes, No. 1. 139 pp.

Autor.https://www.miteco.gob.es/es/biodiversidad/temas/ecosistemas-yconectividad/prescripciones pasos vallados 2a edicion tcm30-195791.pdf

Olson, J.C., Marcarelli, A.M., Timm, A.L.,Eggert, S.L. yKolka, R.K. (2017). Evaluating the Effects of Culver Designs on Ecosystem Processesin Northern Wisconsin Streams.River Researchand Applications.33: 777-787

10.1002/rra.3121 https://www.fs.fed.us/nrs/pubs/irnl/2017/nrs 2017 olson-j 001.pdf

Schall, J. D., Thompson, P. L., Zerges, S. M., Kilgore, R. T., y Morris, J. L. (2012). Hydraulic Design of Highway Culverts.(3th. ed.). FHWA-HIF-12-026.Federal Highway Administration (FHWA).https://www.fhwa.dot.gov/engineering/hydraulics/pubs/12026/hif12026.pdf

United States Department of Agriculture. Forest Service (USFS, 2008). Stream simulation: An ecological approach to provide passage for aquatic organisms at road stream crossing. U.S. Forest Service. Forest Service Simulation Working Group (FSSWG).Author. https://www.fs.fed.us/eng/pubs/pdf/StreamSimulation/hi res/\%20FullDoc.pdf

Vermont Fish and Wildlife Department (VFWD, 2016). Vermont Stream Crossing Handbook. Vermont Fish and Wildlife Department. Author https://vtfishandwildlife.com/sites/fishandwildlife/files/documents/Learn\%20More/Library/REP ORTS\%20AND\%20DOCUMENTS/AOP/AOP\%20HANDBOOK.pdf

\section{SEMBLANZA DEL AUTOR}

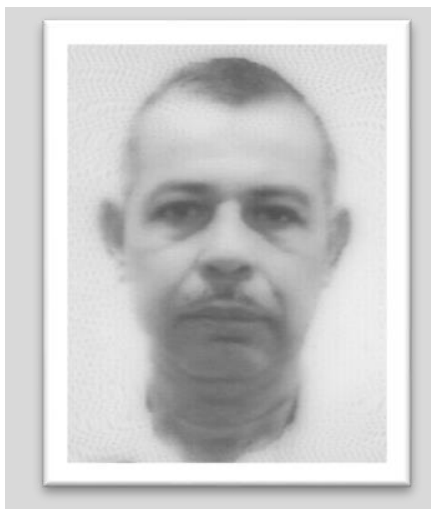

Miguel Enrique Blanco Chávez: Ingeniero Civil, con maestría en Ingeniería Ambiental. Trabaja en el Programa de Investigación de Estudios Nacionales y Servicios del Ambiente (PIENSA) de la UNI. Además, es docente universitario por más de 30 años. 